\title{
COMPLEXITY FOR INFINITE WORDS ASSOCIATED WITH QUADRATIC NON-SIMPLE PARRY NUMBERS*
}

\author{
L'UBOMÍRA BALKOVÁ
}

Communicated by Jean-Pierre Gazeau

\begin{abstract}
Studying of complexity of infinite aperiodic words, i.e., the number of different factors of the infinite word of a fixed length, is an interesting combinatorial problem. Moreover, investigation of infinite words associated with $\beta$-integers can be interpreted as investigation of one-dimensional quasicrystals. In such a way of interpretation, complexity corresponds to the number of local configurations of atoms.
\end{abstract}

\section{Introduction}

To study the structure of an infinite word $u$ on a finite alphabet $\mathcal{A}$ and to measure the diversity of patterns occurring in this word, it is useful to define complexity of $u$. It is a function $C(n)$ which with every $n \in \mathbb{N}$ associates the number of different words of length $n$ contained in $u$. The simplest infinite word is a constant sequence $z^{\omega}$ with $z \in \mathcal{A}$. There exists only one word of each length, therefore $C(n)=1$ for all $n \in \mathbb{N}$. One extreme of the opposite side is a random sequence for which, almost surely, the complexity $C(n)=(\# \mathcal{A})^{n}$. Between these two extremes, one can find infinite eventually periodic words for which the complexity $C(n) \leq n$ for all $n \in \mathbb{N}$, and the simplest aperiodic words, called Sturmian words, with the complexity $C(n)=n+1$ for all $n \in \mathbb{N}$.

Some kinds of infinite aperiodic words can serve as models for one dimensional quasicrystals, i.e., materials with long-range orientational order and sharp diffraction images of non-crystallographic symmetry. To understand the physical properties of these materials, it is important to describe their combinatorial properties. For instance, complexity corresponds to the number of local configurations of atoms.

${ }^{*}$ This paper is a part of the Proceedings of the XXIV Workshop on Geometric Methods in Physics, held in Białowieza, Poland from June 26 - July 2, 2005. 
In this paper, we focus on infinite words $u_{\beta}$ associated with $\beta$-integers $\mathbb{Z}_{\beta}$. It can be shown that for $\beta$ being a Pisot number, i.e., $\beta>1$ being an algebraic integer such that all its Galois conjugates have modulus strictly less than one, $\mathbb{Z}_{\beta}$ is a selfsimilar uniformly discrete and relatively dense set, with self-similarity factor $\beta$ $\left(\beta \mathbb{Z}_{\beta} \subset \mathbb{Z}_{\beta}\right)$. Moreover, it satisfies $\mathbb{Z}_{\beta}-\mathbb{Z}_{\beta} \subset \mathbb{Z}_{\beta}+F$ for a finite set $F \subset \mathbb{R}$. In other words, it is a Delone set [3] fulfilling the Meyer property [4], thus it models a one-dimensional quasicrystal. Recall that if $\beta$ is a Pisot number, then its Rényi expansion of unity (defined in Section 2.1) is eventually periodic, i.e., $\beta$ is a Parry number [7]. Therefore we will concentrate on Parry numbers. Complexity of infinite words associates with simple Parry numbers (numbers with a finite Rényi expansion of unity) has been investigated in [2]. Here, the main attention is devoted to description of complexity of the infinite aperiodic word $u_{\beta}$ being the fixed point of the substitution $\varphi(0)=0^{a} 1, \varphi(1)=0^{b} 1, a \geq b+1$, associated with the Rényi expansion of unity in base $\beta$, where $\beta$ is a quadratic non-simple Parry number.

\section{Notations and Definitions}

An alphabet $\mathcal{A}$ is a finite set of symbols called letters. A concatenation of letters is a word. The set $\mathcal{A}^{*}$ of all finite words (including the empty word $\varepsilon$ ) provided with the operation of concatenation is a free monoid. The length of a word $w=$ $w_{1} w_{2} \ldots w_{n}$ is denoted by $|w|=n$. We will deal also with infinite words $v=$ $v_{1} v_{2} v_{3} \ldots$. A finite word $w$ is called a factor of the word $u$ (finite or infinite) if there exist a finite word $w^{(1)}$ and a word $w^{(2)}$ (finite or infinite) such that $v=$ $w^{(1)} w w^{(2)}$. The word $w$ is a prefix of $u$ if $w^{(1)}=\varepsilon$. Analogically, $w$ is a suffix of $u$ if $w^{(2)}=\varepsilon$. A concatenation of $k$ letters $z$ (or $k$ words $z$ ) will be denoted by $z^{k}$, a concatenation of infinitely many letters $z$ (or words $z$ ) by $z^{\omega}$. An infinite word $v$ is said to be eventually periodic if there exist words $w, z$ such that $v=w z^{\omega}$. Let $v=v_{1} v_{2} v_{3} \ldots$, then $v_{1}^{-1} v=v_{2} v_{3} \ldots$ A factor $w$ of $v$ is called a left special factor of $v$ if there exist distinct letters $y, z \in \mathcal{A}$ such that $y w, z w$ are factors of $v$. We call $y, z$ left extensions of $w$. Similarly for right special factors. Complexity of a word $u$ is a function $C: \mathbb{N} \rightarrow \mathbb{N}$ such that

$$
C(n)=\text { the number of different factors of } u \text { of length } n \text {. }
$$

We will denote by $L(u)$ (language on $u$ ) the set of all factors of a word $u$. A substitution on $\mathcal{A}^{*}$ is a morphism $\varphi: \mathcal{A}^{*} \rightarrow \mathcal{A}^{*}$ such that there exists at least one letter $z \in \mathcal{A}$ satisfying $|\varphi(z)|>1$ and $\varphi(z) \neq \varepsilon$ for all $z \in \mathcal{A}$. Since a morphism satisfies $\varphi(v w)=\varphi(v) \varphi(w)$ for all $v, w \in \mathcal{A}^{*}$, it suffices to define the 
substitution on the alphabet $\mathcal{A}$. An infinite word $u$ is said to be a fixed point of the substitution $\varphi$ if it fulfills

$$
u=u_{1} u_{2} u_{3} \ldots=\varphi\left(u_{1}\right) \varphi\left(u_{2}\right) \varphi\left(u_{3}\right) \ldots=\varphi(u) .
$$

Relation (2) implies that $\varphi^{n}\left(u_{1}\right)$ is a prefix of $u$ for every $n \in \mathbb{N}$ and its length grows with growing $n$. Formally written

$$
u=\lim _{n \rightarrow \infty} \varphi^{n}\left(u_{1}\right) .
$$

Definition 1. A substitution $\varphi$ over the alphabet $\mathcal{A}$ is called primitive if there exists $k \in \mathbb{N}$ such that for any $z \in \mathcal{A}$ the word $\varphi^{k}(z)$ contains all the letters of $\mathcal{A}$.

Definition 2. An infinite word $u$ is called uniformly recurrent if for every $n \in \mathbb{N}$ exists $R(n)>0$ such that any factor of $u$ of length $\geq R(n)$ contains all the factors of $u$ of length $n$.

It can be proved that if $u$ is a fixed point of a primitive substitution $\varphi$, then $u$ is uniformly recurrent [6].

\subsection{Beta-expansions and Beta-integers}

Let $\beta>1$ be a real number and let $x$ be a positive real number. Any convergent series of the form

$$
x=\sum_{i=-\infty}^{k} x_{i} \beta^{i}
$$

where $x_{i} \in \mathbb{N}$, is called a $\beta$-representation of $x$. As well as it is usual for the decimal system, we will denote the $\beta$-representation of $x$ by

$$
\langle x\rangle_{\beta}=x_{k} x_{k-1} \ldots x_{0} \bullet x_{-1} \ldots
$$

if $k \geq 0$, otherwise

$$
\langle x\rangle_{\beta}=0 \bullet \underbrace{0 \ldots \ldots .0}_{(-1-k)-\text { times }} x_{-1} \ldots .
$$

If a $\beta$-representation ends with infinitely many zeros, it is said to be finite and the ending zeros are omitted.

If $\beta \notin \mathbb{N}$, for a given $x$ there can exist more $\beta$-representations. A representation of $x$ can be obtained by the following greedy algorithm: There exists $k \in \mathbb{Z}$ such that 
$\beta^{k} \leq x<\beta^{k+1}$. Let $x_{k}:=\left\lfloor\frac{x}{\beta^{k}}\right\rfloor$ and $r_{k}:=\left\{\frac{x}{\beta^{k}}\right\}$, where $\lfloor$.$\rfloor denotes the lower$ integer part and $\{$.$\} denotes the fractional part. For i<k$, put $x_{i}:=\left\lfloor\beta r_{i+1}\right\rfloor$ and $r_{i}:=\left\{\beta r_{i+1}\right\}$. The representation obtained by the greedy algorithm is called $\beta$ expansion of $x$ and the coefficients of a $\beta$-expansion satisfy: $x_{k} \in\{1, \ldots,\lceil\beta\rceil-1\}$ and $x_{i} \in\{0, \ldots,\lceil\beta\rceil-1\}$ for all $i<k$, where $\lceil$.$\rceil denotes the upper integer$ part. We will use for $\beta$-expansion of $x$ the notation $\langle x\rangle_{\beta}$. If $x=\sum_{i=-\infty}^{k} x_{i} \beta^{i}$ is the $\beta$-expansion of a nonnegative number $x$, then $\sum_{i=-\infty}^{-1} x_{i} \beta^{i}$ is called the $\beta$-fractional (or simply fractional) part of $x$. Let us introduce some important notions connected with $\beta$-expansions:

- The set of nonnegative numbers with vanishing fractional part are called nonnegative $\beta$-integers, formally

$$
\mathbb{Z}_{\beta}^{+}:=\left\{x \geq 0 \mid\langle x\rangle_{\beta}=x_{k} x_{k-1} \ldots x_{0} \bullet\right\} .
$$

- The set of $\beta$-integers is then defined by

$$
\mathbb{Z}_{\beta}:=-\mathbb{Z}_{\beta}^{+} \cup \mathbb{Z}_{\beta}^{+}
$$

The Rényi expansion of unity simplifies description of elements of $\mathbb{Z}_{\beta}$. For its definition, we introduce the transformation $T_{\beta}(x):=\{\beta x\}$ for $x \in[0,1]$. The Rényi expansion of unity in base $\beta$ is defined as

$$
d_{\beta}(1)=t_{1} t_{2} t_{3} \ldots \quad \text { where } \quad t_{i}:=\left\lfloor\beta T_{\beta}^{i-1}(1)\right\rfloor .
$$

Every number $\beta>1$ is characterized by its Rényi expansion of unity. Note that $t_{1}=\lfloor\beta\rfloor \geq 1$. Not every sequence of nonnegative integers is equal to $d_{\beta}(1)$ for some $\beta$. Parry studied this problem in his paper [5]: A sequence $\left(t_{i}\right)_{i \geq 1}, t_{i} \in \mathbb{N}$, is the Rényi expansion of unity for some number $\beta$ if and only if the sequence satisfies

$$
t_{j} t_{j+1} t_{j+2} \ldots \prec t_{1} t_{2} t_{3} \ldots \quad \text { for every } j>1
$$

where $\prec$ denotes strictly lexicographically smaller.

The Rényi expansion of unity enables us to decide whether a given $\beta$-representation of $x$ is the $\beta$-expansion or not. For this purpose, we define the infinite Rényi expansion of unity

$$
d_{\beta}^{*}(1)= \begin{cases}d_{\beta}(1) & \text { if } d_{\beta}(1) \quad \text { is infinite } \\ \left(t_{1} t_{2} \ldots t_{m-1}\left(t_{m}-1\right)\right)^{\omega} & \text { if } d_{\beta}(1)=t_{1} \ldots t_{m} \text { with } t_{m} \neq 0\end{cases}
$$

Parry has proved also the following proposition. 
Proposition 3. Let $d_{\beta}^{*}(1)$ be an infinite Rényi expansion of unity. Let $\sum_{i=-\infty}^{k} x_{i} \beta^{i}$ be a $\beta$-representation of a positive number $x$. Then $\sum_{i=-\infty}^{k} x_{i} \beta^{i}$ is a $\beta$-expansion of $x$ if and only if $x_{i} x_{i-1} \ldots \prec d_{\beta}^{*}(1)$ for all $i \leq k$.

\subsection{Infinite Words Associated with Beta-integers}

If $\beta$ is an integer, then clearly $\mathbb{Z}_{\beta}=\mathbb{Z}$ and the distance between neighboring elements of $\mathbb{Z}_{\beta}$ for a fixed $\beta$ is always one. The situation changes dramatically if $\beta \notin \mathbb{N}$. In this case, the number of different distances between neighboring elements of $\mathbb{Z}_{\beta}$ is at least two. In [8], it is shown that the distances occurring between neighbors of $\mathbb{Z}_{\beta}$ form the set $\left\{\Delta_{k} \mid k \in \mathbb{N}\right\}$, where

$$
\Delta_{k}:=\sum_{i=1}^{\infty} \frac{t_{i+k}}{\beta^{i}} \quad \text { for } \quad k \in \mathbb{N} .
$$

It is evident that the set $\left\{\Delta_{k} \mid k \in \mathbb{N}\right\}$ is finite if and only if $d_{\beta}(1)$ is eventually periodic.

When $d_{\beta}(1)$ is eventually periodic, we will call $\beta$ a Parry number. When $d_{\beta}(1)$ is finite, it is said to be a simple Parry number. Every Pisot number, i.e., a real algebraic integer greater than 1 , all of whose conjugates are of modulus strictly less than one, is a Parry number.

From now on, we will restrict our considerations to quadratic Parry numbers. The Rényi expansion of unity for a simple quadratic Pisot number $\beta$ is equal to $d_{\beta}(1)=a b$, where $a \geq b$. Hence, $\beta$ is exactly the positive root of the polynomial $x^{2}-a x-b$. Whereas the Rényi expansion of unity for a non-simple quadratic Pisot number $\beta$ is equal to $d_{\beta}(1)=a b^{\omega}$, where $a>b \geq 1$. Consequently, $\beta$ is the greater root of the polynomial $x^{2}-(a+1) x+a-b$. Drawn on the real line, there are only two distances between neighboring points of $\mathbb{Z}_{\beta}$. The longer distance is always $\Delta_{0}=1$, the smaller one is $\Delta_{1}$. Conversely, if there are exactly two types of distances between neighboring points of $\mathbb{Z}_{\beta}$ for $\beta>1$, then $\beta$ is a quadratic Pisot number.

If we assign the numbers 0 and 1 to the two types of distances $\Delta_{0}$ and $\Delta_{1}$, respectively, and write down the order of distances in $\mathbb{Z}_{\beta}^{+}$on the real line, we naturally obtain an infinite word; we will denote this word by $u_{\beta}$. Since $\beta \mathbb{Z}_{\beta}^{+} \subset \mathbb{Z}_{\beta}^{+}$, it can be shown easily that the word $u_{\beta}$ is a fixed point of a certain substitution $\varphi$ (see e.g. [2]). In particular, for the simple quadratic Pisot number $\beta$, the generating substitution is

$$
\varphi(0)=0^{a} 1, \quad \varphi(1)=0^{b}
$$


for the non-simple quadratic Pisot number $\beta$, the generating substitution is

$$
\varphi(0)=0^{a} 1, \quad \varphi(1)=0^{b} 1 .
$$

\section{Complexity of $u_{\beta}$ Associated with $d_{\beta}(1)=a b^{\omega}$}

We have found inspiration for determination of complexity in the paper [2] where the complexity of a large class of simple Parry numbers is determined. In order to determine complexity of the infinite word $u_{\beta}$ being the fixed point of the substitution $\varphi(0)=0^{a} 1, \varphi(1)=0^{b} 1$, we will use the following proposition.

Proposition 4. Let us denote by $M_{n}$ the set of all left special factors of $L\left(u_{\beta}\right)$ of length $n$. Then the first difference of the complexity is

$$
C(n+1)-C(n)=\# M_{n} .
$$

Proof: Every word $v \in L\left(u_{\beta}\right)$ can be viewed as $v=z u$ where $u \in L\left(u_{\beta}\right)$ and $z \in\{0,1\}$. Therefore the complexity function does not increase for words $u$ which have a unique left extension. Apparently, every left special word of length $n$ contributes to the increase of complexity by one. Consequently, $C(n+1)-$ $C(n)=\# M_{n}$.

To find the exact values of $C(n)$, it suffices to find all the left special factors of length $n$. For this purpose, let us define some useful notions.

Definition 5. A left special factor $v \in L\left(u_{\beta}\right)$ is called maximal if neither $v 0$ nor $v 1$ are left special.

Definition 6. An infinite word $u$ is called an infinite left special factor of $u_{\beta}$ if each prefix of $u$ is a left special factor of $L\left(u_{\beta}\right)$.

Example 7. Let us illustrate a few of left special factors of

$$
u_{\beta}=0001000100010100010001000101 \ldots
$$

being the fixed point of the substitution $\varphi(0)=0001, \varphi(1)=01$ by construction of the head of a tree containing left special factors. Beginning from the empty word to the right, one can read all left special factors of length $n \in\{1,2, \ldots, 14\}$. There are two maximal left special factors 00, 01000100010 having length $<14$. 


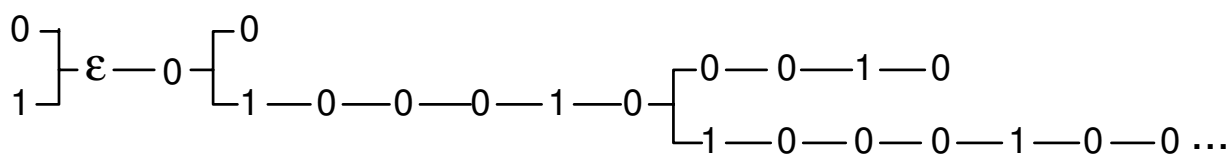

Since every left special factor is a prefix of a maximal or an infinite left special factor, our aim is to investigate all maximal left special factors and all infinite left special factors of $L\left(u_{\beta}\right)$.

If $b=a-1$, then $\beta$ is the larger root of the polynomial $x^{2}-(a+1) x+1$, i.e., $\beta$ is a unit. For quadratic Pisot units, it has been shown in [1] that $C(n)=n+1$, i.e., the corresponding word is Sturmian. Consequently, it suffices to consider the case of $1 \leq b<a-1$.

We will introduce lemmas which enable to determine the form of maximal and infinite left special factors.

Lemma 8. Every left special factor that contains at least one 1 has the prefix $0^{b} 1$. Left special factors which do not contain 1 and are not maximal have the form $0^{r}$, $r<0^{a-1}$. The maximal left special factor which does not contain 1 has the form $0^{a-1}$.

Lemma 9. For every left special factor $v$ which has the suffix 1 , there exists a left special factor $w$ such that $v=0^{b} 1 \varphi(w)$.

Proof: The existence of such $w \in L\left(u_{\beta}\right)$ is obvious. It suffices to show that $w$ is left special. Since we can find both $0 v$ and $1 v$ in $L\left(u_{\beta}\right)$, we have $0 v=0^{b+1} 1 \varphi(w)$, then necessarily $0^{a} 1 \varphi(w)=\varphi(0 w) \in L\left(u_{\beta}\right)$, and $1 v=10^{b} 1 \varphi(w)=1 \varphi(1 w)$, hence, $w$ is a left special factor of $L\left(u_{\beta}\right)$.

In order to determine complexity, we need to study the so-called total bispecial factors.

Definition 10. A factor $v$ of $u_{\beta}$ is called total bispecial if both $v 0$ and $v 1$ are left special factors of $u_{\beta}$.

Lemma 11. Let $w \in L\left(u_{\beta}\right)$ and let us denote by $T(w)=0^{b} 1 \varphi(w) 0^{b}$. Then, $w$ is a left special factor if and only if $T(w)$ is a left special factor. Moreover, $w$ is maximal if and only if $T(w)$ is maximal and $w$ is total bispecial if and only if $T(w)$ is total bispecial. 
Proof: Let $w$ be left special, then $0 w, 1 w \in L\left(u_{\beta}\right) . T(0 w)=0^{b} 10^{a} 1 \varphi(w) 0^{b}$, hence $0 T(w) \in L\left(u_{\beta}\right) . T(1 w)=0^{b} 10^{b} 1 \varphi(w) 0^{b}$, thus $1 T(w) \in L\left(u_{\beta}\right)$.

If $T(w)$ is left special, then $0 T(w), 1 T(w) \in L\left(u_{\beta}\right)$. Consequently, $0^{a} 1 \varphi(w) 0^{b}=$ $\varphi(0 w) 0^{b} \in L\left(u_{\beta}\right)$ and $10^{b} 1 \varphi(w) 0^{b}=1 \varphi(1 w) 0^{b} \in L\left(u_{\beta}\right)$, i.e., $0 w, 1 w \in L\left(u_{\beta}\right)$. If $w$ is maximal, then neither $w 0$, nor $w 1$ is left special. Suppose that $T(w)$ is not maximal, then either $T(w) 0$ or $T(w) 1$ is left special. Either $0^{b} 1 \varphi(w) 0^{a} 1$ is left special, hence $w 0$ is left special, which is a contradiction. Or, $0^{b} 1 \varphi(w) 0^{b} 1$ is left special, thus $w 1$ is left special, which is a contradiction, too.

If $T(w)$ is maximal, then neither $T(w) 0$, nor $T(w) 1$ is left special. Suppose that $w$ is not maximal, then either $w 0$ or $w 1$ is left special. Either $0^{b} 1 \varphi(w) 0^{a} 10^{b}$ is left special, hence $T(w) 0$ is left special, which is a contradiction. Or, $0^{b} 1 \varphi(w) 0^{b} 10^{b}$ is left special, thus $T(w) 1$ is left special, which is a contradiction, too.

Analogically for total bispecial factors.

Lemma 12. Let $v, w \in L\left(u_{\beta}\right)$ such that $v$ is a prefix of $w$. Then, $T(v)$ is a prefix of $T(w)$.

Using Lemma 11 and Lemma 12, we can describe the form of maximal and total bispecial factors.

Corollary 13. All maximal left special factors have the form

$$
U^{(1)}=0^{a-1}, \quad U^{(n)}=T\left(U^{(n-1)}\right)=0^{b} 1 \varphi\left(U^{(n-1)}\right) 0^{b} \quad \text { for } n \geq 2 .
$$

All total bispecial factors have the form

$$
V^{(1)}=0^{b}, \quad V^{(n)}=T\left(V^{(n-1)}\right)=0^{b} 1 \varphi\left(V^{(n-1)}\right) 0^{b} .
$$

Moreover, $V^{(n-1)}$ is a prefix of $V^{(n)}$ and $V^{(n)}$ is a prefix of $U^{(n)}$ for all $n \in \mathbb{N}$.

Lemma 14. There exists one infinite left special factor of the form $\lim _{n \rightarrow \infty} V^{(n)}$.

Proof: Each prefix of $\lim _{n \rightarrow \infty} V^{(n)}$ is a prefix of $V^{(k)}$ for some $k \in \mathbb{N}$, therefore it is a left special factor. Assume that there are more infinite left special factors. Let us choose $v^{(1)}, v^{(2)}$ such that $d\left(v^{(1)}, v^{(2)}\right):=\min \left\{k \mid v_{k}^{(1)} \neq v_{k}^{(2)}\right\}$ is minimal. Then there exist infinite left special factors $w^{(1)}, w^{(2)}$ such that $v^{(1)}=0^{b} 1 \varphi\left(w^{(1)}\right)$ and $v^{(2)}=0^{b} 1 \varphi\left(w^{(2)}\right)$. Necessarily, $d\left(w^{(1)}, w^{(2)}\right)<d\left(v^{(1)}, v^{(2)}\right)$ which is a contradiction. 
We know that every left special factor $w$ is either a prefix of a maximal left special factor or a prefix of an infinite left special factor. For $n$ such that

$$
\left|V^{(k)}\right|<n \leq\left|U^{(k)}\right| \text { for some } \quad k \in \mathbb{N}
$$

there exist two left special factors of length $n$. The values $\left|V^{(k)}\right|,\left|U^{(k)}\right|$ play an essential role for determining of complexity. Let us derive their values.

\subsection{Lengths of $V^{(k)}, U^{(k)}$}

Lemma 15. Let us denote by $\left|V^{(n)}\right|_{0}$ the number of Os of the total bispecial factor $V^{(n)}$ and by $\left|V^{(n)}\right|_{1}$ the number of 1 s of $V^{(n)}$. Then $\left|V^{(n)}\right|=\left|V^{(n)}\right|_{0}+\left|V^{(n)}\right|_{1}$ and it holds

$$
\left|V^{(1)}\right|_{0}=b, \quad\left|V^{(1)}\right|_{1}=0, \quad\left(\begin{array}{l}
\left|V^{(n+1)}\right|_{0} \\
\left|V^{(n+1)}\right|_{1}
\end{array}\right)=\left(\begin{array}{cc}
a & b \\
1 & 1
\end{array}\right)\left(\begin{array}{l}
\left|V^{(n)}\right|_{0} \\
\left|V^{(n)}\right|_{1}
\end{array}\right)+\left(\begin{array}{c}
2 b \\
1
\end{array}\right) .
$$

Proof: Let us remind the recursive definition of $V^{(n)}$ :

$$
V^{(1)}=0^{b}, \quad V^{(n+1)}=0^{b} 1 \varphi\left(V^{n)}\right) 0^{b} .
$$

As the substitution considered is $\varphi(0)=0^{a} 1, \varphi(1)=0^{b} 1$, one can see that if we know the values of $\left|V^{(n)}\right|_{0},\left|V^{(n)}\right|_{1}$, then

$$
\left|V^{(n+1)}\right|_{0}=b+a\left|V^{(n)}\right|_{0}+b\left|V^{(n)}\right|_{1}+b, \quad\left|V^{(n+1)}\right|_{1}=1+\left|V^{(n)}\right|_{0}+\left|V^{(n)}\right|_{1} \text {. }
$$

Lemma 16. Let us denote by $\left|U^{(n)}\right|_{0}$ the number of 0 s of the maximal left special factor $U^{(n)}$ and by $\left|U^{(n)}\right|_{1}$ the number of 1 s of $U^{(n)}$. Then $\left|U^{(n)}\right|=\left|U^{(n)}\right|_{0}+$ $\left|U^{(n)}\right|_{1}$ and it holds

$$
\left|U^{(1)}\right|_{0}=a-1, \quad\left|U^{(1)}\right|_{1}=0, \quad\left(\begin{array}{l}
\left|U^{(n+1)}\right|_{0} \\
\left|U^{(n+1)}\right|_{1}
\end{array}\right)=\left(\begin{array}{cc}
a & b \\
1 & 1
\end{array}\right)\left(\begin{array}{l}
\left|U^{(n)}\right|_{0} \\
\left|U^{(n)}\right|_{1}
\end{array}\right)+\left(\begin{array}{c}
2 b \\
1
\end{array}\right) .
$$

Proof: Analogical to the proof of Lemma 15.

At this moment, we have gained enough information to determine complexity $u_{\beta}$ associated with $d_{\beta}(1)=a b^{\omega}, a-1>b$.

Theorem 17. Let $u_{\beta}$ be the fixed point of the substitution $\varphi(0)=0^{a} 1, \varphi(1)=$ $0^{b} 1$. Then for all $n \in \mathbb{N}$

$$
\triangle C(n)=C(n+1)-C(n)=\left\{\begin{array}{cc}
2 & \left|V^{(k)}\right|<n \leq\left|U^{(k)}\right| \text { for } a k \in \mathbb{N} \\
1 & \text { otherwise }
\end{array}\right.
$$


Then complexity can be calculated by

$$
C(n)=\sum_{j=1}^{n-1} \triangle C(j)+C(1)=\sum_{j=1}^{n-1} \triangle C(j)+2 .
$$

Proof: Using Proposition 4, we have $\triangle C(n)=$ the number of left special factors of length $n$ in $L\left(u_{\beta}\right)$. There is one left special factor of length $n$ being a prefix of the infinite left special factor. Since $\left|V^{(k)}\right|<\left|U^{(k)}\right|<\left|V^{(k+1)}\right|$ and $V^{(k)}$ is a prefix of $U^{(k)}$, then there exists a left special factor of length $n$ being prefix of $U^{(k)}$ and not prefix of $V^{(k)}$ if $\left|V^{(k)}\right|<n \leq\left|U^{(k)}\right|$. Figure 1 illustrates the tree of left special factors for $u_{\beta}$ being the fixed point of the substitution $\varphi(0)=$ $0001, \varphi(1)=01$. We can see total bispecial factors $V^{(k)}$ and maximal left special factors $U^{(k)}$ for $k=1,2$.

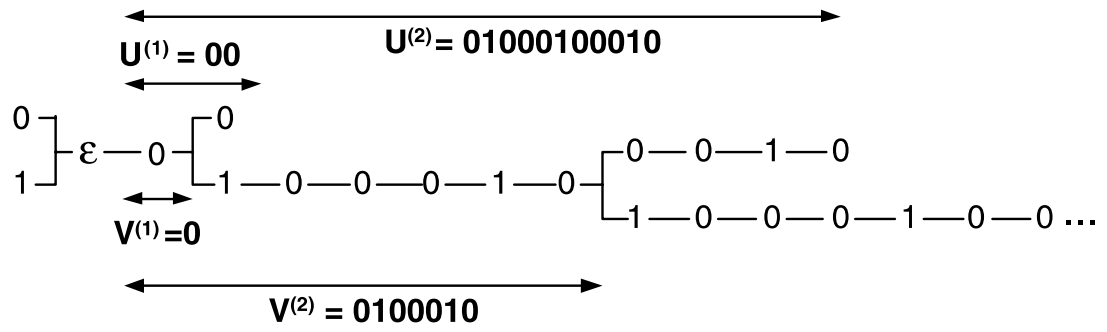

Figure 1.

\section{Conclusion}

Studying of complexity of infinite aperiodic words is an interesting combinatorial problem. Moreover, investigation of infinite words associated with $\beta$-integers $\mathbb{Z}_{\beta}$ can be interpreted as investigation of one-dimensional quasicrystals. In this paper, we have considered infinite words $u_{\beta}$ associated with $\mathbb{Z}_{\beta}$ for $\beta$ being a quadratic algebraic integer corresponding to an eventually periodic Rényi expansion of unity in base $\beta$. We have investigated its complexity using methods which can be applied for any infinite aperiodic words obtained by substitution. This paper together with the study of simple Parry numbers [2] builds up a complete investigation of complexity of infinite aperiodic words connected with quadratic Parry numbers. 


\section{References}

[1] Burdík Č., Frougny Ch., Gazeau J.-P. and Krejcar R., $\beta$-integers as Natural Counting Systems for Quasicrystals, J. Phys. A 31 (1998) 6449-6472.

[2] Frougny Ch., Masáková Z. and Pelantová E., Complexity of Infinite Words Associated with $\beta$-expansions, Theor. Appl. 38 (2004) 163-185.

[3] Lagarias J., Geometric Models for Quasicrystals I. Delone Sets of Finite Type, Discrete Comput. Geom. 21 (1999) 161-191.

[4] Meyer Y., Quasicrystals, Diophantine Approximation, and Algebraic Numbers, In: Beyond Quasicrystals, F. Axel and D. Gratias (Eds.), Les Houches, Springer, Berlin, 1995, pp 3-16.

[5] Parry W., On the $\beta$-expansions of Real Numbers, Acta Math. Acad. Sci. Hungar. 11 (1960) 401-416.

[6] Queffélec M., Substitution Dynamical Systems-Spectral Analysis, Lecture Notes in Mathematics 1294, Springer, 1987.

[7] Schmidt K., On Periodic Expansions of Pisot Numbers and Salem Numbers, Bull. London Math. Soc. 12 (1980) 269-278.

[8] Thurston W., Groups, Tilings, and Finite State Automata, Geometry Supercomputer Project Research Report GCG1, University of Minnesota, 1989.

L’ubomíra Balková

Department of Mathematics

Czech Technical University in Prague

Prague, CZECH REPUBLIC

E-mail address: 1.balkova@centrum.cz 